\title{
Epidemiology of Needlestick Injuries Among Healthcare Workers in Tehran, Iran: A Cross-Sectional Study
}

\author{
Hossein Masoumi-Asl, ${ }^{1,2}$ Mohammad Rahbar, ${ }^{2}$ Anoushirvan Soltani, ${ }^{2}$ Zahra Pezeshki, ${ }^{1}$ hadijeh \\ Khanaliha, ${ }^{3, *}$ and Goodarz Kolifarhood ${ }^{4}$ \\ ${ }^{1}$ Center for Communicable Diseases Control, Ministry of Health and Medical Education, Tehran, Iran \\ ${ }^{2}$ Center for Clinical Researches, Milad Hospital, Tehran, Iran \\ ${ }^{3}$ Research Center of Pediatric Infectious Diseases, Rasoul-e-Akram Hospital, Iran University of Medical Sciences, Tehran, IR Iran \\ ${ }^{4}$ Department of Epidemiology, School of Public Health, Shahid Beheshti University of Medical Sciences,Tehran, Iran \\ "Corresponding author: Khadijeh Khanaliha, Research Center of Pediatric Infectious Diseases, Rasoul-e-Akram Hospital, Iran University of Medical Sciences, Tehran, IR Iran. \\ Fax:+98-2166516049, E-mail: khanalihakh@yahoo.com
}

Received 2016 March 05; Revised 2016 October 26; Accepted 2016 November 06.

\begin{abstract}
Background: Healthcare workers (HCW) such as medical, dental, nursing and midwifery workers are at high risk for occupational exposure to blood-borne pathogens such as hepatitis B virus (HBV), hepatitis C virus (HCV) and HIV through sharp injuries of needlestick.

Objectives: The current study aimed to evaluate the prevalence of needlestick injuries (NSIs) among healthcare workers in Milad hospital in Tehran, Iran, from 2007 to 2011 and investigate the related factors of needle sticking in the hospital in order to decrease the risk of infectious diseases transmission due to needlestick injury.

Methods: This retrospective cross-sectional study was performed among healthcare workers including nursing staff, physicians, gynecologist, laboratory staff, cleaners and garbage collectors in Milad hospital in Tehran, Iran, over a five-year period from 2007 to 2011. All of the NSIs data was obtained from infection control committee in Milad hospital. Questionnaire sheets were filled up and all of the information was rechecked one more time by experts. The information included demographic questions and NSI related questions. Vaccination for HBV and titer of hepatitis B surface antibody (anti-HBs) were recorded. The association between demographic and needlestick characteristics was tested through working shifts.

Results: In the current study, 608 out of 3806 hospital staff including 209(34.3\%) male and 399 (65.7\%) female had NSIs over a five-year period in Milad hospital. Most of the NSIs was observed in enrolled nurse, nursing sister and midwife groups 308 (50.7\%) followed by servants 122 (20.1\%) and the lowest was found in laboratory staff 14 (2.3\%). Staff who worked in the internal medicine ward 95 (15.6\%), emergency ward 72 (11.8\%) and intensive care unit (ICU) 58 (9.5\%) had experienced NSIs. There were uniform time patterns of NSIs by working shifts; therefore, the lowest number of the injuries occurred in the start of working shift (7:00 am, 01:00 pm and 07:00 pm) and increased along the time. Most of the NSIs occurred through taking blood and injection 182 (29.9\%). Compared to NSI data by working shift, the greater number of the injuries occurred in the evening and night, but it was not statistically significant through needlestick characteristics ( $P$ value $>0.05$ ).

Conclusions: Nurses are at the highest risk for NSIs and servants the second. Staff training, proper use of protective equipment, proper disposal of infectious waste and vaccination of all staff against HBV might be effective measures towards reduction of the number of NSIs.
\end{abstract}

Keywords: Health Personnel, Hospitals, Needlestick Injuries

\section{Background}

Accidental needlestick injuries (NSIs) are a hazard for healthcare workers such as nurses, physicians and other hospital workers (1). The national institute of occupational safety and health (NIOSH) of USA defines needlestick injuries as injuries caused by needles such as hypodermic needles, blood collection needles, intravenous (IV) stylets and needles used to connect parts of IV delivery systems (2). Healthcare workers (HCW) such as medical, dental, nursing and midwifery workers are at high risk for occupa- tional exposure to blood-borne pathogens such as hepatitis B virus (HBV), hepatitis C virus (HCV) and HIV through sharp injuries of needlestick $(1,3,4)$, whatever the average risk of being infected with HIV from occupational exposure is estimated to be low in comparison with HBV or HCV $(5,6)$. The other infections which can transmit through needlesticks are syphilis, malaria and herpes (7). Factors that determine an HCW's risk of infection include the frequency of needlestick incidents, the pathogen involved, the immune status of the worker, the severity of the NSI and use of appropriate post-exposure prophylaxis (PEP)

Copyright ( ) 2016, Infectious Diseases and Tropical Medicine Research Center. This is an open-access article distributed under the terms of the Creative Commons Attribution-NonCommercial 4.0 International License (http://creativecommons.org/licenses/by-nc/4.0/) which permits copy and redistribute the material just in noncommercial usages, provided the original work is properly cited. 
(8). According to the world health organization (WHO), $16000 \mathrm{HCV}, 66000 \mathrm{HBV}$ and 1000 cases of HIV may have occurred worldwide in the year 2000 among HCWs through their exposure to NSIs (9). Approximately three million healthcare workers receive an injury with an occupational instrument annually, with around 2000000 exposures to HBV and 1000000 to HCV. Almost 75\% of the total occupational exposure is percutaneous and only $25 \%$ is mucosalcutaneous. The infecting risk of HBV or HCV in a healthcare worker is higher in percutaneous than mucosal-cutaneous exposure (4). Hepatitis C virus infection is one of the most common chronic blood borne infections that cause chronic liver disease. The prevalence of hepatitis B core antibodies (anti-HBc) among hepatitis B surface antigen (HBsAg) negative blood donors was $9.98 \%$ in Tehran, Iran, in 2014 (10). The risk of infection after a single needlestick exposure to infected blood average is $1.8 \%$ for $\mathrm{HCV}$ (11) and no vaccine is available to prevent $\mathrm{HCV}$ infection (12). About 300 million persons worldwide are carriers of the HBV and about $0.41 \%$ of blood-donors in Iran were HBsAg positive in 2007 (13). Seroconversion after a single needlestick exposure to an HBV-infected patient is estimated 6\% - 30\% (11). HIV affects more than 36 million persons worldwide (11) .The risk of transmission of HIV infections to the healthcare worker by needlestick injuries is about $0.3 \%$ (11). Moreover, transmission risk depends on the viral load of the patient and the amount of blood that passes from one to the other (14). The NSIs among different groups of HCWs are reported from Iran $(3,15-18)$.

The evaluation of NSIs prevalence among different groups of health care workers and related factors is very important to implement prevention programs and precautions to decrease blood born infections.

\section{Objectives}

The current study aimed to evaluate prevalence of NSIs among health care workers in Milad Hospital in Tehran, Iran, and investigate related factors of needle-sticking in the hospital in order to decrease the transmission risk of infectious diseases due to needlestick injury.

\section{Methods}

The current cross-sectional research was performed among healthcare workers including nursing staff, physicians, gynecologist, laboratory staff and workers in Milad hospital in Tehran, Iran, over a five-year period from 2007 to 2011. Milad hospital is the largest specialized and subspecialized hospital in Tehran. This hospital is a complementary health service provider in Iran's social security organi- zation (SSO) chain of hospitals. It has 1000 beds, 24 surgery rooms, 20 pre-surgery and 20 post-surgery care beds.

The healthcare workers were a vast number of practitioners from various specialty areas including surgery, emergency and internal medicine wards, intensive care unit, obstetrics and gynecology, and pediatrics wards and laboratory. This study was approved by the ethics committee of Milad hospital (ethic no. 91/2153) in accordance with Helsinki declaration and guidelines. All of NSIs information was obtained from infection control committee in $\mathrm{Mi}$ lad hospital. All of the healthcare workers in the morning, evening and night shifts with full information from 2007 to 2011 were included and the ones with inadequate information were excluded from the study.

Questionnaire sheets were filled up and all of the information was rechecked one more time by experts. The information included demographic questions and NSI related questions, gender, age, years of professional experience, education level, existence/not existence of safety box, recapping/un-recapping of needles, number and time of NSIs, the action resulting in NSIs, using/not using latex gloves, contamination/non-contamination of exposed needles with patients' blood or other discharges of patients with HBV, HCV and HIV infections, kind of injury, predisposing reason of NSIs, vaccination against HBV and titer of hepatitis B surface antibody (anti-HBs) and post injury prophylaxis.

Statistical analysis: The association between demographic and needlestick characteristics was tested through working shifts (morning and evening-night) using Chi-square test by SPSS version 18 .

\section{Results}

Table 1 shows demographic characteristics of healthcare workers by working shift. In the current study, 608 (15.9\%) out of 3806 hospital staff, including 209 (34.3\%) male and 399 (65.7\%) female, had NSIs in medical history over a five-year period in Milad hospital. The incidence of NSIs was $2.9 \%-3.5 \%$ annually.

The age range of staff was $19-55$ years. The highest frequency of NSIs 243 (39.9\%) was found in 26 - 30 year old group and the lowest frequency of NSIs $8(1.3 \%)$ belonged to 51 - 55 year old group (Table 1). Most of the NSIs were observed in enrolled nurse, nursing sister and midwife group 308 (50.7\%) followed by cleaners and garbage collectors (servants) $122(20.1 \%$ ) and the lowest was found in laboratory staff 14 (2.3\%) (Table 1). Compared to NSIs data regarding working shift, greater number of the injuries occurred in the evening and night but it was not statistically significant based on staff's demographic characteristics ( $P$ value $>0.05$ ). Moreover, healthcare workers who worked 
Table 1. Frequency of Demographic Characteristics of Staff Who Had NSIs Based on Working Shifts ( $=608)$

\begin{tabular}{|c|c|c|c|}
\hline \multirow[t]{2}{*}{ Demographic Characteristics } & \multicolumn{2}{|c|}{ Working Shift } & \multirow[b]{2}{*}{ P Value } \\
\hline & Morning, $N=285(46.9 \%)$ & Evening-Night, $\mathrm{N}=323(53.1 \%)$ & \\
\hline Age group & & & 0.417 \\
\hline $19-25$ & $20(7 \%)$ & $32(9.9 \%)$ & \\
\hline $26-30$ & $112(39.3 \%)$ & $131(40.6 \%)$ & \\
\hline $36-40$ & $42(14.7 \%)$ & $39(12.1 \%)$ & \\
\hline $41-45$ & $26(9.1 \%)$ & $17(5.3 \%)$ & \\
\hline $46-50$ & $7(2.5 \%)$ & $7(2.2 \%)$ & \\
\hline \multirow[t]{2}{*}{$51-55$} & $4(1.4 \%)$ & $4(1.2 \%)$ & \\
\hline & Chi-square $=6.06$ & & \\
\hline Gender & & & 0.307 \\
\hline Male & $92(32.3 \%)$ & $117(36.2 \%)$ & \\
\hline \multirow[t]{2}{*}{ Female } & $193(67.7 \%)$ & $206(63.8 \%)$ & \\
\hline & Chi-square $=1.04$ & & \\
\hline Occupation status & & & 0.09 \\
\hline Enrolled nurse, nursing sister, midwife & $135(47.4 \%)$ & $173(53.6 \%)$ & \\
\hline Anesthesia personnel/surgical physicians & $62(22.1 \%)$ & $51(15.8 \%)$ & \\
\hline General internal physicians & $10(3.5 \%)$ & $5(1.5 \%)$ & \\
\hline Laboratory staffs & $9(3.2 \%)$ & $5(1.5 \%)$ & \\
\hline Servants & $53(18.2 \%)$ & $69(21.4 \%)$ & \\
\hline Radiology, secretary and guard & $16(5.6 \%)$ & $20(6.2 \%)$ & \\
\hline
\end{tabular}

in internal medicine ward 95 (15.6\%), emergency ward 72 (11.8\%) and intensive care unit (ICU) 58 (9.5\%) had experienced NSIs in the current study (data not shown).

Table 2 indicates the needlestick characteristics by working shift. Although most NSIs happened during taking blood and injection 182 (29.9\%) and in surgery 71 (11.6\%), the frequency of NSIs was remarkably high during cleaning up and garbage removing 106 (17.4\%). The most damaged organ was hand 510 (83.9\%), followed by foot 28 (4.6\%), the commonest non-needlestick exposure was eyes with 61 (10\%) splashed with bloody fluid. The NSIs in most cases was found by using needle 471 (77.5\%) during surgery, taking blood and injection; however, in some cases was observed by using sharp objects such as lancet. Deep wound was made in 466 (76.6\%) and superficial wound in 42 (6.9\%) and mild deep wound in 15 (2.5\%) as a kind of NSIs depth. In total $43(7.1 \%)$ out of 608 reported NSIs, the needles were contaminated from cases who had HBV, HCV and HIV infections. Furthermore, 32 (5.3\%) of cases were source of HBV, 10 (1.6\%) HCV and 1 (0.2\%) HIV (Table 2). In the cur- rent study 430 (70.7\%) of NSIs were immunized for HBV, 2 (0.32\%) were not immunized and 176 (28.9\%) were not confirmed by receiving $\mathrm{HBV}$ vaccine in formal documentation. The NSIs were reported in 405(66.6\%) who had antibody against $\mathrm{HBV} \geq 10 \mathrm{IU} / \mathrm{mL}, 44$ (7.2\%) had antibody $\leq 10 \mathrm{IU} / \mathrm{mL}$ and about 159(26.1\%) of NSIs had uncertain situation. Compared to NSI data by working shift, the greater number of the injuries occurred in the evening and night shifts but it was not statistically significant through needlestick characteristics (P value $>0.05$ ) (Table 2 ).

The frequency of NSIs from 2007 to 2011 is summarized in Table 3. The highest frequency of NSIs was found in 2007 and the lowest in 2009 among staff of Milad hospital. There was no significant difference between years and frequency of NSIs in the study. The highest frequency of NSIs occurred in spring 174 (28.6\%). Most of the NSIs were reported during 9:00 - 12:00 am, with the highest frequency at 9:00 am (67). Also, there were uniform time patterns of NSIs by working shifts; therefore, the lowest number of the injuries occurred in the start of working shift (7:00 am, 01:00 pm and 
Table 2. Needlestick Characteristics of Healthcare Workers Based on Working Shift $(\mathrm{N}=608)$

\begin{tabular}{|c|c|c|c|}
\hline \multirow[t]{2}{*}{ Needlestick Characteristics } & \multicolumn{2}{|c|}{ Working Shift } & \multirow[t]{2}{*}{ PValu } \\
\hline & Morning, $N=285(46.9 \%)$ & Evening-Night, $\mathrm{N}=323(53.1 \%)$ & \\
\hline Causes of needlestick injury & & & 0.370 \\
\hline Taking blood and injection & $88(30.9 \%)$ & $94(29.1 \%)$ & \\
\hline Recapping needle & $6(2.1 \%)$ & $11(3.4 \%)$ & \\
\hline Surgery & $37(13 \%)$ & $34(10.5 \%)$ & \\
\hline Cleaning/collecting garbage & $42(4.7 \%)$ & $64(19.8 \%)$ & \\
\hline \multirow[t]{2}{*}{ Other activities } & $112(39.3 \%)$ & $120(37.2 \%)$ & \\
\hline & Chi-square $=4.27$ & & \\
\hline Damaged organ & & & 0.458 \\
\hline Hand & $238(83.5 \%)$ & $272(84.2 \%)$ & \\
\hline Foot & $11(3.9 \%)$ & $17(5.3 \%)$ & \\
\hline Eye splashing & $33(11.6 \%)$ & $28(8.7 \%)$ & \\
\hline \multirow[t]{2}{*}{ Other organ } & $3(1.1 \%)$ & $6(1.9 \%)$ & \\
\hline & Chi-square $=2.59$ & & \\
\hline Contaminants & & & 0.6 \\
\hline Blood & $277(97.2 \%)$ & $313(96.9 \%)$ & \\
\hline Body liquids & $7(2.5 \%)$ & $6(1.9 \%)$ & \\
\hline \multirow[t]{2}{*}{ Uncertain } & $1(0.4 \%)$ & $4(1.2 \%)$ & \\
\hline & Chi-square $=1.02$ & & \\
\hline Sharp objects & & & 0.745 \\
\hline Needle & $217(76.1 \%)$ & $254(78.6 \%)$ & \\
\hline Lancet & $19(6.7 \%)$ & $19(5.9 \%)$ & \\
\hline Others & $12(4.2 \%)$ & $16(5 \%)$ & \\
\hline \multirow[t]{2}{*}{ Uncertain } & $37(13 \%)$ & $34(10.5 \%)$ & \\
\hline & Chi-square $=1.23$ & & \\
\hline Depth of wounds & & & 0.288 \\
\hline Superficial wound & $23(8.1 \%)$ & $19(5.9 \%)$ & \\
\hline Mild deep wound & $5(1.8 \%)$ & $10(3.1 \%)$ & \\
\hline Deep wound & $212(74.4 \%)$ & $254(78.6 \%)$ & \\
\hline \multirow[t]{2}{*}{ Uncertain } & $45(15.8 \%)$ & $40(12.4 \%)$ & \\
\hline & Chi-square $=3.76$ & & \\
\hline Known source of needle contamination & & & 0.981 \\
\hline HBV & $16(5.6 \%)$ & $16(5 \%)$ & \\
\hline $\mathrm{HCV}$ & $5(1.8 \%)$ & $5(1.5 \%)$ & \\
\hline HIV & 0 & $1(0.3 \%)$ & \\
\hline \multirow[t]{2}{*}{ Uncertain } & $264(92.6 \%)$ & $301(93.2 \%)$ & \\
\hline & Chi-square $=1.06$ & & \\
\hline HBV vaccination & & & 0.686 \\
\hline Yes & $207(72.6 \%)$ & $223(69 \%)$ & \\
\hline No & $1(0.4 \%)$ & $1(0.3 \%)$ & \\
\hline \multirow[t]{2}{*}{ Uncertain } & $77(27 \%)$ & $99(30.7)$ & \\
\hline & Chi-square $=1.21$ & & \\
\hline HBV antibody level & & & 0.227 \\
\hline Antibody $\geq 10 \mathrm{IU} / \mathrm{mL}$ & $188(66 \%)$ & $217(67.2 \%)$ & \\
\hline Antibody $\leq 10 \mathrm{IU} / \mathrm{mL}$ & $16(5.6 \%)$ & $28(8.7 \%)$ & \\
\hline \multirow[t]{2}{*}{ Uncertain } & $81(28.4 \%)$ & $78(24.1 \%)$ & \\
\hline & Chi-square $=3.04$ & & \\
\hline
\end{tabular}

Abbreviations: $\mathrm{HBV}$, hepatitis B virus; $\mathrm{HCV}$, hepatitis $\mathrm{C}$ virus. 
07:00 pm) and increased along the time (Figure 1).

\section{Discussion}

Healthcare workers (HCWs) are subject to exposures to hazardous blood borne pathogens; therefore, follow-up is necessary (19). In the current study, 608 hospital staff had NSIs in medical practice from 2007 to 2011 in Milad Hospital, Tehran, Iran. The results of the current survey showed that the lowest and highest rates of NSIs were 112 (18.3\%) and 132 (21.7\%) over a five-year period. The highest rate of NSIs was observed in the 26-35 year old age group. Healthcare staff who worked in internal medicine ward 95 (15.6\%), emergency ward 72 (11.8\%) and ICU 58 (9.5\%) had experienced NSIs in the current study and most of the NSIs were observed in nursing group 308 (50.7\%); cleaners and garbage collectors (servants) were the second 122 (20.1\%). Most of the NSIs were reported during 9:00 - 12:00 am. In the current study, the highest frequency was at 9:00 am.

The NSIs among nurses was reported more than the other at risk groups in some previous studies $(15-18,20)$. The results of a study showed that the incidence of needle injuries among nursing workers was $45.12 \%$ during their working period and occupational injuries caused by sharp objects and needles and angiocath needle had caused the most serious injuries with a frequency of $43.91 \%$ and $29.05 \%$, respectively. Most of the injuries occurred in the emergency ward (48.64\%) and in terms of working shifts, the injuries were $15.54 \%, 29.72 \%$ and $54.72 \%$ in the morning, evening and night shifts, respectively (16). In the present study, more NSIs occurred in the evening and night shifts but the difference was statistically insignificant.

The NSIs among health care workers were reported 175 (49.3\%) of 355 healthcare personnel in Tehran. The NSIs were higher in nurses $(54.9 \%$ ) compared to the physicians (36.7\%) (17).

A Study in Shiraz reported 71.1\% NSIs in medical, dental, nursing and midwifery students at the university hospitals of Shiraz (3). A study was conducted on 158 nursing workers of Khanevadeh Hospital in Tehran to study NSIs. The results of this study showed that $56.96 \%$ of nurses had history of at least one NSI and $22.15 \%$ of them had NSIs during the last year. Injections were the most common actions due to NSIs (24.44\%) and recapping of needles was in the second place (21.11\%), operating room had the highest prevalence $(18.9 \%)$ of needlestick injuries among all wards of the hospital; emergency ward and ICU followed(15.6\%) (18). The prevalence of NSIs in Iranian nurses was also high (52.6\%) in Fars province of Iran (20), which were consistent with the results of the current study; 50.7\% NSIs among nurses and taking blood and injection as the most common action due to NSIs, however in the current study in- ternal medicine had the highest NSIs experience 95 (15.6\%), emergency 72 (11.8\%) and ICU 58 (9.5\%) followed. In contrast to the current study, in another study NSIs were high among residents in training (76\%) and only $11 \%$ in nurses (21). The NSIs rate is reported in $47 \%$ of healthcare workers including $52 \%$ of physicians and $40 \%$ of nurses in Jamaica and rates of occupational exposure were $74.3 \%, 61.1 \%, 51.9 \%$ and $51 \%$ in residents, laboratory technicians, nurses and interns, respectively in Tehran (22).

Another study reported that re-capping needles accounted for $(21 \%)$ of injuries, various minor procedures (21\%), injury during surgery (19.4\%) and taking blood (12\%) (23). Taking blood and injection $182(29.9 \%)$ were the most vulnerable procedures associated with NSIs in healthcare workers in the current study, followed by clean-up and garbage working activities 106 (17.4\%) and NSIs during surgery 71 (11.7\%), which indicates needles without recapping or neglect using safety box to collect the garbage and exposing workers to NSIs risk factors cause NSIs among healthcare workers due to inappropriate implementation of safe injection and using safety boxes to remove sharp objects.

The commonest clinical activities that caused the NSIs were blood withdrawal and recapping needles in some studies $(20,24-26)$ and the most related factors were work experience and use of gloves and education (26-28).

The frequency of NSIs between cleaners and garbage collectors was 122 (20.1\%) in the current study. This is the most important finding in the study in comparison with those of the other studies (Table 1). In 471 (77.5\%) cases, needle was the source of NSIs and in $38(6.2 \%)$ cases it was related to other sharp objects; 466 (76.6\%) of them had experienced deep wound in this study that showed the staff were careless. The location of damaged organs, especially the hands and fingers account for $83.9 \%$ of the cases. Splattering blood and other potentially infectious materials to eye occurred in $10 \%$ of the cases and foot harm caused by sharp syringes indicates that disposal of medical waste containing syringes damages feet of garbage collectors. In $7 \%$ of cases it was confirmed that the source of contaminated needles was the patients with HBV, HCV and HIV; however in $93 \%$ of cases, the person or the source of contamination was not confirmed in the present study. Also, $70.7 \%$ of people with NSIs had received hepatitis B vaccination, $0.32 \%$ had not been vaccinated and vaccination of $28.9 \%$ of people was not registered in formal sheets; $74 \%$ of those with NSIs had been evaluated for hepatitis B antibodies and higher antibody titer of $10 \mathrm{IU} / \mathrm{mL}$ was observed in $66.6 \%$ of them; therefore, it is recommended to take effective measures to prevent infections from occupational exposure of healthcare workers to blood including immunization against HBV, eliminating unnecessary injections, 
Table 3. The Frequency of NSIs Among Healthcare Workers

\begin{tabular}{|c|c|c|c|c|c|}
\hline \multirow[t]{2}{*}{ Year } & \multirow[b]{2}{*}{ Spring } & \multicolumn{3}{|c|}{ Season } & \multirow[t]{2}{*}{ Total } \\
\hline & & Summer & Autumn & Winter & \\
\hline 2007 & 38 & 27 & 29 & 38 & $132(21.7)$ \\
\hline 2008 & 36 & 26 & 38 & 19 & $119(19.4)$ \\
\hline 2009 & 34 & 31 & 24 & 23 & $112(18.3)$ \\
\hline 2010 & 37 & 35 & 19 & 37 & $128(20.9)$ \\
\hline 2011 & 29 & 29 & 26 & 33 & $117(19.1)$ \\
\hline Total (\%) & $174(28.6)$ & $148(24.3)$ & $136(22.3)$ & $150(24.5)$ & $608(100)$ \\
\hline
\end{tabular}

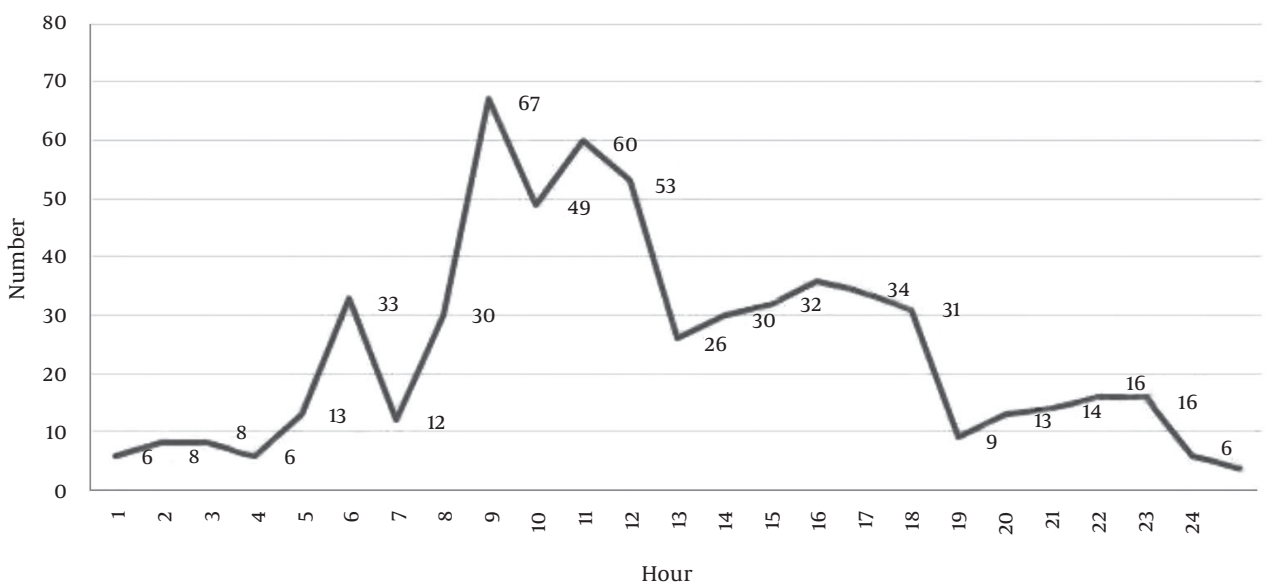

Figure 1. Frequency of NSIs, According to Working Shift (Hours) Among Healthcare Workers from 2007 to 2011 in Milad Hospital, Tehran

implementing universal precautions, eliminating needle recapping and disposing of the sharp in to a sharps container immediately after use, utilize safer devices such as needles that sheath or retract after use. NSIs post-exposure activities including notifying safety office, consulting doctors, identification of source of infection and follow up the situation of NSIs in health worker are necessary. Obviously, training health workers on the dangers of NSIs is also important.

In The current study, needlestick injuries were evaluated among healthcare workers in Milad Hospital in Tehran, Iran, over a five-year period and the related factors of needlesticking. There were uniform time patterns of NSIs by working shifts and the lowest number of the injuries occurred in the start of working shift and increased in time. Compared to NSI data by working shift, the greatest numbers of the injuries occurred in the evening and night but the difference was not statistically significant. A limitation of this study was the lack of some information, which was retrospectively collected.

\subsection{Conclusion}

Checking the items of NSIs over a five-year period in Milad hospital showed that nurses were the group with the highest risk and cleaners and garbage collectors followed. Most NSIs lesions with the needle occurred in the evening and night shifts and the injuries were deep. Staff training, proper use of protective equipment, proper disposal of infectious waste, using safety box and vaccinating all staff against $\mathrm{HBV}$ can reduce the number and effects of the NSIs threats.

\section{Acknowledgments}

The current study (Project No: 92/1649) was supported by center for clinical researches of Milad hospital. The authors would like to appreciate the members of infection control committee and center for clinical researches of $\mathrm{Mi}$ lad hospital. 


\section{Footnote}

Conflict of Interest: The authors declared no conflict of interest regarding the materials and results of the current study.

\section{References}

1. Atenstaedt RL, Payne S, Roberts RJ, Russell IT, Russell D, Edwards RT. Needle-stick injuries in primary care in Wales. J Public Health (Oxf). 2007;29(4):434-40. doi: 10.1093/pubmed/fdm048. [PubMed 17998261]

2. Norsayani MY, Noor Hassim I. Study on incidence of needle stick injury and factors associated with this problem among medical students. J Occup Health. 2003;45(3):172-8. [PubMed:14646293].

3. Askarian M, Malekmakan L. The prevalence of needle stick injuries in medical, dental, nursing and midwifery students at the university teaching hospitals of Shiraz, Iran. Indian J Med Sci. 2006;60(6):227-32. [PubMed: 16790948].

4. Coppola N, De Pascalis S, Onorato L, Calo F, Sagnelli C, Sagnelli E. Hepatitis $B$ virus and hepatitis $C$ virus infection in healthcare workers. World J Hepatol. 2016;8(5):273-81. doi: 10.4254/wjh.v8.i5.273. [PubMed: 26925201].

5. Mohammadi N, HassanpourDehkordi A, NikbakhatNasrabadi A. Iranian patients with chronic hepatitis struggle to do self-care. Life Sci J. 2013;10(1):457-62.

6. hassanpour dehkordi A. The concept of self-care in patients diag nosed with chronic hepatitis: A rogers evolutionary approach. JUrmia Nurs Midwifery Fac. 2014;12(6):477-90.

7. Control CfD Prevention. . Guidelines for infection control in healthcare personnel. Am J Infect Control. 1998;26:289-354.

8. Alert NIOSH. Preventing needlestick injuries in health care settings. DHHS (NIOSH) Publication. 1999:2000-108.

9. Pruss-Ustun A, Rapiti E, Hutin Y. Estimation of the global burden of disease attributable to contaminated sharps injuries among health-care workers. Am J Ind Med. 2005;48(6):482-90. doi: 10.1002/ajim.20230. [PubMed: 16299710].

10. Alizadeh Z, Milani S, Sharifi Z. Occult hepatitis B virus infection among Iranian blood donors: a preliminary study. Archives Iran Med (AIM). 2014;17(2).

11. Newsom DH, Kiwanuka JP. Needle-Stick Injuries. Bandolier Extra: Evidence based Healthcare. ; 2003.

12. Safety NIfO Health. . NIOSH Alert: Preventing needle-stick injuries in healthcare setting. National Institute for Occupation Safety and HealthDHHS publication; .

13. Kafi-abad SA, Rezvan H, Abolghasemi H. Trends in prevalence of hepatitis B virus infection among Iranian blood donors, 1998-2007. Transfus Med. 2009;19(4):189-94. doi: 10.1111/j.1365-3148.2009.00935.x. [PubMed: 19708860].
14. Elmiyeh B, Whitaker IS, James MJ, Chahal CA, Galea A, Alshafi K. Needlestick injuries in the National Health Service: a culture of silence. JR Soc Med. 2004;97(7):326-7. doi:10.1258/jrsm.97.7.326. [PubMed: 15229257].

15. Motaarefi H. Factors associated with needlestick injuries in health care occupations: A systematic review. J Clinical Diagnostic Res. 2016 doi: $10.7860 / j \mathrm{cdr} / 2016 / 17973.8221$.

16. Ehsani SR, Mohammadnejad E, Hadizadeh MR, Mozaffari J, Ranjbaran S, Deljo R, et al. Epidemiology of needle sticks and sharp injuries among nurses in an Iranian teaching hospital. Arch Clin Infect Dis. 2012;8(1):27-30.

17. Shiva F, Sanaei A, Shamshiri AR, Ghotbi F. Survey of needle-stick injuries in paediatric health personnel of 5 university hospitals in Tehran. J Pak Med Assoc. 2011;61(2):127-31. [PubMed: 21375158].

18. Galougahi MH. Evaluation of needle stick injuries among nurses of Khanevadeh Hospital in Tehran. Iran J Nurs Midwifery Res. 2010;15(4):172-7. [PubMed: 21589791].

19. Panlilio AL, Cardo DM, Grohskopf LA, Heneine W, Ross CS. Updated US Public Health Service guidelines for the management of occupational exposures to HIV and recommendations for postexposure prophylaxis. US Department of Health and Human Services, Centers for Disease Control and Prevention. 2005.

20. Askarian M, Shaghaghian S, McLaws ML. Needlestick injuries among nurses of Fars province, Iran. Ann Epidemiol. 2007;17(12):988-92. doi: 10.1016/j.annepidem.2007.07.106. [PubMed:17923420].

21. Rele M, Mathur M, Turbadkar D. Risk of needle stick injuries in health care workers - a report. Indian J Med Microbiol. 2002;20(4):206-7. [PubMed: 17657071].

22. Farsi D, Zare MA, Hassani SA, Abbasi S, Emaminaini A, Hafezimoghadam $P$, et al. Prevalence of occupational exposure to blood and body secretions and its related effective factors among health care workers of three emergency departments in Tehran. J Res Med Sci. 2012;17(7):656

23. Foster TM, Lee MG, McGaw CD, Frankson MA. Prevalence of needlestick injuries and other high risk exposures among healthcare workers in Jamaica. West Indian Med J. 2010;59(2):153-8. [PubMed: 21275118].

24. Rodrigues C. Needle stick injuries \& the health care worker-the time to act is now. Indian J Med Res. 2010;131:384-6. [PubMed: 20418551].

25. Ashat M, Bhatia V, Puri S, Thakare M, Koushal V. Needle stick injury and HIV risk among health care workers in North India. Indian J Med Sci. 2011;65(9):371-8. doi: 10.4103/0019-5359.108947. [PubMed: 23508479].

26. Afridi AA, Kumar A, Sayani R. Needle stick injuries-risk and preventive factors: a study among health care workers in tertiary care hospitals in Pakistan. Glob J Health Sci. 2013;5(4):85-92. doi: 10.5539/gjhs.v5n4p85. [PubMed: 23777725].

27. Gourni P, Polikandrioti M, Vasilopoulos G, Mpaltzi E, Gourni M. Occupational Exposure to blood and body fluids of nurses at Emergency department. Health Sci J. 2012;6(1)

28. Parsa-Pili J, Izadi N, Golbabaei F. Factors associated with needle stick and Sharp injuries among health care workers. Inter J Occupational Hygiene. 2015;5(4):191-7. 